C. Owen Lovejoy, et al.

Science 326, 70 (2009);

DOI: $10.1126 /$ science. 1175827

The following resources related to this article are available online at www.sciencemag.org (this information is current as of October 14, 2009 ):

Updated information and services, including high-resolution figures, can be found in the online version of this article at:

http://www.sciencemag.org/cgi/content/full/326/5949/70

Supporting Online Material can be found at:

http://www.sciencemag.org/cgi/content/full/326/5949/70/DC1

A list of selected additional articles on the Science Web sites related to this article can be found at:

http://www.sciencemag.org/cgi/content/full/326/5949/70\#related-content

This article cites $\mathbf{4 3}$ articles, 11 of which can be accessed for free:

http://www.sciencemag.org/cgi/content/full/326/5949/70\#otherarticles

This article has been cited by 6 articles hosted by HighWire Press; see:

http://www.sciencemag.org/cgi/content/full/326/5949/70\#otherarticles

This article appears in the following subject collections:

Anthropology

http://www.sciencemag.org/cgi/collection/anthro

Information about obtaining reprints of this article or about obtaining permission to reproduce this article in whole or in part can be found at:

http://www.sciencemag.org/about/permissions.dtl 


\title{
Careful Climbing in the Miocene: The Forelimbs of Ardipithecus ramidus and Humans Are Primitive
}

\author{
C. Owen Lovejoy, Scott W. Simpson, Tim D. White, Berhane Asfaw, Gen Suwa
}

A grasping hand and highly mobile forelimb are defining characteristics of primates. The special ability to pick things up and manipulate them has probably been a central selective force in making primates so unusually intelligent. It's something that porpoises can't do at all and crows can't do very well. It may also be one reason why humans alone eventually evolved cognition.

The hands of African apes are specialized in a number of ways that make them dramatically different from our own. Apes must support their large body mass during climbing to feed and nest, especially in the middle and higher parts of the tree canopy. Their hands must therefore withstand very high forces, and this is facilitated by their elongated palms and fingers. Our palms are much shorter and our wrists more mobile. This allows us to grasp objects and compress them with great dexterity and force - something often called a "power grip." The differences between ape and human forelimbs become less pronounced going from the hand to the shoulder. Ape and human elbow joints, for example, diverge only moderately in their manner of load transmission.

The high loads that apes bear during locomotion have required them to greatly stiffen the joints between their fingers and palms. Because their thumb has not been elongated in the same way as their palms and fingers have, thumb-to-palm and thumb-to-finger oppositions are more awkward for them. We are therefore much more adept at making and using tools. All of these forelimb characteristics in apes have led them to adopt an unusual form of terrestrial quadrupedality, in which they support themselves on their knuckles rather than on their palms. Only African apes exhibit this "knuckle-walking." Other primates, such as monkeys, still support themselves on their palms.

It has long been assumed that our hands must have evolved from hands like those of African apes. When they are knuckle-walking, their long forelimbs angle their trunks upward. This posture has therefore long been viewed by some as "preadapting" our ancestors to holding their trunks upright.

Until now, this argument was unsettled, because we lacked an adequate fossil record. Even Lucy, the most complete Australopithecus skeleton yet found, had only two hand bones - far short of the number needed to interpret the structure and evolution of the hand. The Ardipithecus skeleton reported here changes that. Not only is it more than 1 million years older than Lucy (4.4 million versus 3.2 million years old), its hands are virtually complete and intact. They show that Ardipithecus did not knuckle-walk like African apes and that it lacked virtually all of the specializations that protect great ape hands from injury while they climb and feed in trees.

Ardipithecus hands were very different from those of African apes. Its wrist joints were not as stiff as those of apes, and the joints between their palms and fingers were much more flexible. Moreover, a large joint in the middle of the wrist (the midcarpal joint) was especially flexible, being even more mobile than our own. This would have allowed Ardipithecus to support nearly all of its body weight on its palms when moving along tree branches, so that it could move well forward of a supporting forelimb without first releasing its grip on a branch.

This discovery ends years of speculation about the course of human evolution. Our ancestors' hands differed profoundly from those of living great apes, and therefore the two must have substantially differed in the ways they climbed, fed, and nested. It is African apes who have evolved so extensively since we shared our last common ancestor, not humans or our immediate hominid ancestors. Hands of the earliest hominids were less ape-like than ours and quite different from those of any living form.

Ardipithecus also shows that our ability to use and make tools did not require us to greatly modify our hands. Rather, human grasp and dexterity were long ago inherited almost directly from our last common ancestor with chimpanzees. We now know that our earliest ancestors only had to slightly enlarge their thumbs and shorten their fingers to greatly improve their dexterity for tool-using.

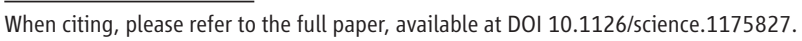




\section{Careful Climbing in the Miocene: The Forelimbs of Ardipithecus ramidus and Humans Are Primitive}

\author{
C. Owen Lovejoy, ${ }^{1 *}$ Scott W. Simpson, ${ }^{2}$ Tim D. White, ${ }^{3 *}$ Berhane Asfaw, ${ }^{4}$ Gen Suwa ${ }^{5}$
}

The Ardipithecus ramidus hand and wrist exhibit none of the derived mechanisms that restrict motion in extant great apes and are reminiscent of those of Miocene apes, such as Proconsul. The capitate head is more palmar than in all other known hominoids, permitting extreme midcarpal dorsiflexion. Ar. ramidus and all later hominids lack the carpometacarpal articular and ligamentous specializations of extant apes. Manual proportions are unlike those of any extant ape. Metacarpals 2 through 5 are relatively short, lacking any morphological traits associable with

knuckle-walking. Humeral and ulnar characters are primitive and like those of later hominids. The Ar. ramidus forelimb complex implies palmigrady during bridging and careful climbing and exhibits none of the adaptations to vertical climbing, forelimb suspension, and knuckle-walking that are seen in extant African apes.

$\mathrm{T}$ he grasping hand is a hallmark of all primates (1), and elaborated forelimb flexibility characterizes all extant hominoids. Hands have played a central role in human evolution and perhaps even in the emergence of higher cognition (2). Although we no longer use our hands in locomotion, our ancestors must have. Chimpanzees, which knuckle-walk and vertically climb, have long fingers compared to their thumbs. Gorilla proportions are similar but less extreme. Did our ancestors also knuckle-walk and adapt to vertical climbing and suspension as the modern African apes have, or did our anatomy emerge directly from a more generalized Miocene ancestor, as some mid-20th century anatomists argued (3)? Answers to these central questions are now provided by Ardipithecus ramidus.

The Aramis Ar ramidus sample includes all bones of the forelimb except for the pisiform and some terminal phalanges (4). Of particular importance are $A R A-V P-7 / 2$, a forelimb skeleton, and $A R A-V P-6 / 500$, a partial skeleton preserving a lower arm and most of both hands (Fig. 1). We describe here the salient aspects of the taxon's forelimb anatomy and their implications for hominid evolution. These and other data (5-7) show that $A r$ ramidus was both terrestrially bipedal and arboreally capable.

\footnotetext{
${ }^{1}$ Department of Anthropology, School of Biomedical Sciences, Kent State University, Kent, OH 44240-0001, USA. ${ }^{2}$ Department of Anatomy, Case Western Reserve University School of Medicine, Cleveland, OH 44106-4930, USA. ${ }^{3}$ Human Evolution Research Center, and Department of Integrative Biology, 3101 Valley Life Sciences Building, University of California, Berkeley, CA 94720, USA. ${ }^{4}$ Rift Valley Research Service, Post Office Box 5717, Addis Ababa, Ethiopia. ${ }^{5}$ The University Museum, the University of Tokyo, Hongo, Bunkyo-ku, Tokyo 1130033, Japan.
}

${ }^{*}$ To whom correspondence should be addressed. E-mail: olovejoy@aol.com (C.O.L.); timwhite@berkeley.edu (T.D.W.)
The mating surfaces for the Mc4 and Mc5 bases on the Ar. ramidus hamulus lack the angled and distopalmarly extending facets that are present in Pan, Pongo, and (to a lesser degree) Gorilla. These are additional reflections of restricted mobility and increased rigidity of the Mc4- and Mc5-hamate joints in these apes. The hamate's primitive state (a hamulus still permitting substantial Mc5-hamate dorsiflexion) in Sivapithecus parvada (19), Oreopithecus bambolii, Proconsul sp. (14-17), and E. africanus [(10), this study] implies that a more rigid hamatemetacarpal articulation emerged in parallel between early ancestors of African apes and Pongo. This rigidity was therefore unrelated to knuckle-walking and may have emerged only in large-bodied suspensory forms. Mobility in the Mc5-hamate joint, as seen in Ar. ramidus, facilitates and/or reflects compliance of the palm with the substrate during palmigrady, as well as providing hypothenar opposition to the first ray during grasping (20). The latter proved to be a pivotal exaptation for extractive foraging and eventually tool using and making in both Australopithecus (21) and Homo, but especially in the latter.

The Ar. ramidus phalanges of rays 2 to 5 (figs. S7 to S15 and table S2) are shorter than those of Pan but longer than those of Gorilla, relative to body size. However, because of elongation of the medial metacarpus in African apes, phalangeal-to-metacarpal length ratios in Ar. ramidus are more similar to those of Old World monkeys (figs. S8 and S14). Manual/ pedal phalangeal length ratios are similar in Ar. ramidus, Pan, and Gorilla and are substantially higher than those in Proconsul (fig. S15).

The first ray. Unlike in apes, the Ar. ramidus first ray is relatively large and robust (Fig. 1, figs. S16 to S22, and tables S1 to S3). The Mc1 base flares outward with a prominent attachment for the abductor pollicis longus muscle, as in all later hominids. In both size and proportions, its head is robust and dorsally expanded, with welldefined and asymmetric sesamoid grooves as in Homo but in distinction to Pan or Gorilla. The $A R A-V P-6 / 500 \mathrm{Mc} 1 / \mathrm{Mc} 5$ length ratio is close to that of Proconsul and exceeds those of extant African apes, largely reflecting elongation of the medial metacarpus in the latter (fig. S17).

The first ray's terminal phalanx exhibits a symmetrically constructed, rugose insertion gable $(22,23)$ for the flexor pollicis longus (Fig. 1, inset), in contrast to African apes in which this muscle's tendon is reduced or absent (3). The first ray's carpometacarpal, MP, and interphalangeal joints are also somewhat larger and more humanlike in shape than those of African apes, suggesting greater thenar mobility and/or possibly greater loading during ontogeny [that is, types 1 and/or 4 (18)]. The trapezium's tuberosity is large and projects toward the palm (Fig. 3), deepening the adjacent groove for the flexor carpi radialis tendon. 
The central joint complex and midcarpal joint. Ar. ramidus provides pivotal evidence about the natural history of the immobile (24) or fixed unit (25) of the hand (trapezoid/capitate/ proximal Mc2 and Mc3) that we refer to as the central joint complex (CJC). Most previous analyses have presumed a modern apelike antecedent of the human CJC, without due regard for the key anatomy described below. Ar. ramidus shows that CJC anatomy is pivotal to understanding the evolution of the hominoid hand.
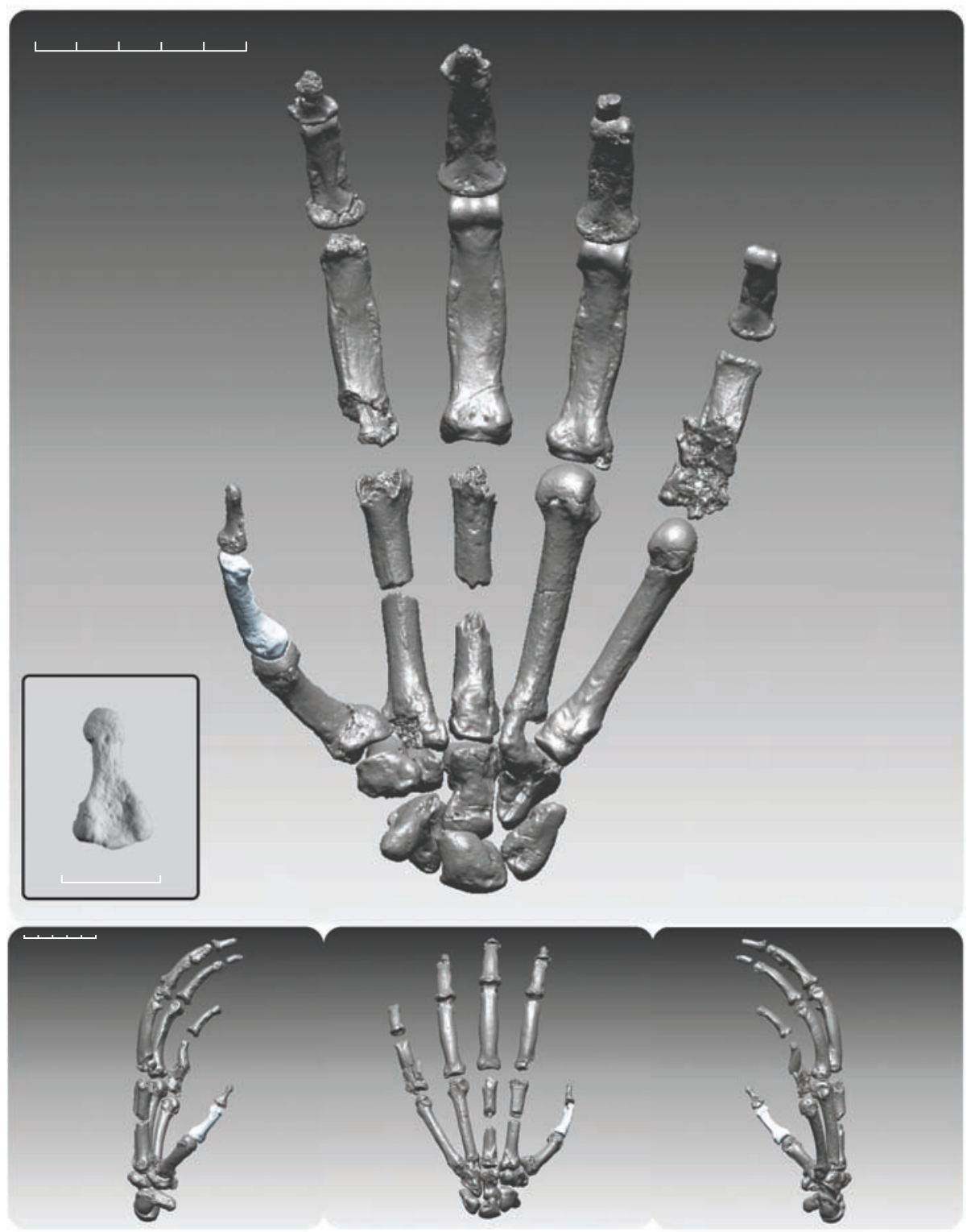

Fig. 1. Digitally rendered composite hand of $A R A-V P-6 / 500$ in palmar view. Lateral (bottom left), dorsal (bottom center), and medial (bottom right) views are also shown. All carpals except for the trapezium are from the left side. The trapezium, Mc2, and some phalanges have been mirror-imaged. The first ray's proximal phalanx is from ARA-VP-7/2 and has been size-adjusted based on estimated Mc4 length (7) in the two specimens (estimated in $A R A-V P-6 / 500$ ). Intermediate and terminal phalanges are provisionally allocated to position and side only, except for ARA-VP-6/500-049, a pollical terminal (inset; cast). Note its clearly marked insertion gable for the flexor pollicis longus as in modern humans. Imagery is based on computed tomography $(\mathrm{CT})$ scans taken at $150-\mu \mathrm{m}$ voxel resolution by a peripheral quantitative computed tomography (pQCT) XCT-Research SA+ instrument (Stratek, Pforzheim, Germany), and processed by use of software Analyze 6.0 (Mayo Clinic, Rochester, MN) and Rapidform 2004/2006 (Inus Technology, Seoul, South Korea). portions of their joint surfaces are angled opposite to their palmar portions (Fig. $5 \mathrm{H}$ ), creating a partial screw effect.

This morphology has several extraordinary advantages. The partial screw effect (26) provides a route for cartilage-sparing energy dissipation during loading. In palmar view (Fig. $5 \mathrm{H}$ ), it can be seen that external axial rotation of the Mc3 upon the capitate causes distention of the joint, which can then be resisted by tension in greatly hypertrophied carpometacarpal ligaments. However, the Mc2 and Mc3 cannot rotate (from a neutral position) in the opposite direction, because such internal rotation is now blocked by the capitate; that is, by the position of the capitate's palmar process, which has displaced the (less stable) trapezoid and abuts against the Mc2.

Finally, the overall proximodorsal-todistopalmar angulation of the Mc3-capitate interface converts any dorsopalmar shear to tension. Thus, dorsopalmar shear and torsion acting in the CJC are both resisted by the greatly enlarged and axially disposed carpometacarpal ligaments (see below and Figs. 3 to 5). The topographically complex (polyaxial) and heavily buttressed African ape CJCs increase rigidity and permit energy dissipation during suspension (Figs. 3 to 5) and vertical climbing (27), and possibly during knuckle-walking.

The CJC of Ar. ramidus is very different. It exhibits the simple, planar joint interface shared with some Old World monkeys and Proconsul sp. (14-17), in which all four CJC elements meet one another at nearly a single dorsopalmar axis (Figs. 3 and 5). This configuration is less competent to restrict torsion and dorsopalmar shear within the CJC, because it can only resist rotation and/or shear via its slight subchondral surface undulations, whose relative translation only minimally distends the joint.

The configuration of CJC joint geometry can be assessed visually (fig. S23) and by two metrics: angulation of the capitate's hamate facet relative to the long axis of the Mc3 (fig. S23) and mediolateral angulation of the Mc3-capitate joint's dorsal surface relative to the Mc3 shaft's axis (figs. S24 and S25). Whereas both metrics record minimal angulation in palmigrade taxa, such as Papio and Ar. ramidus, both are elevated in Pan and Gorilla. However, angulation of this joint is also present in Homo. In the latter, it may have been associated with transfer of the styloid anlage (28) from the capitate to the Mc3, because the transfer had not yet occurred in Ar. ramidus, which also did not yet exhibit any angulation (it is actually slightly negative). Thus, the transfer may have either been directly associated with the introduction of Mc3 ulnar deviation or, alternatively, a pleiotropic consequence of changes in pattern formation underlying it. But it seems quite probable that the introduction of this angulation was to enhance the opposition of the medial rays with the hypertrophied thumb (29).

The development of axial ligaments and their attachment patterns is also an integral part of the 
above CJC functional morphology. In extant great apes, massive centrally located carpometacarpal ligaments pass through deep notches in the capitate and hamate to attach to $\mathrm{Mc} 2$ to $\mathrm{Mc} 4$ (Figs. 3 to 5). Thickening of these CJC ligaments, as indicated by substantial expansion of their passageways from capitate to Mc2 and Mc3, has occurred in virtually all hominoids engaged in suspension, including Pongo, Pan, and Gorilla. Osteologically, such thickening is expressed as isolation of dorsal and palmar intermetacarpal articular facets separated by deep cylindrical grooves.

Whereas D. laietanus exhibits the extant great ape condition, similar capitate and Mc base notching is entirely absent in Proconsul, Ar.

ramidus, Australopithecus, and Homo, reflecting the absence of similarly robust carpometacarpal ligaments in these taxa. The CJC's primitive state in Ar. ramidus strongly suggests that the earliest hominids and their immediate ancestors did not engage in habitual suspension or vertical climbing.

The proximal part of the Ar. ramidus capitate also differs from all known hominoid homologs. Its head and neck lie more palmar to the bone's corpus (Fig. 4), and the head's dorsal articular surface blends distally into a broad dorsal depression that accommodates the distal edges of the scaphoid and lunate during midcarpal joint dorsiflexion (30). In knuckle-walking African

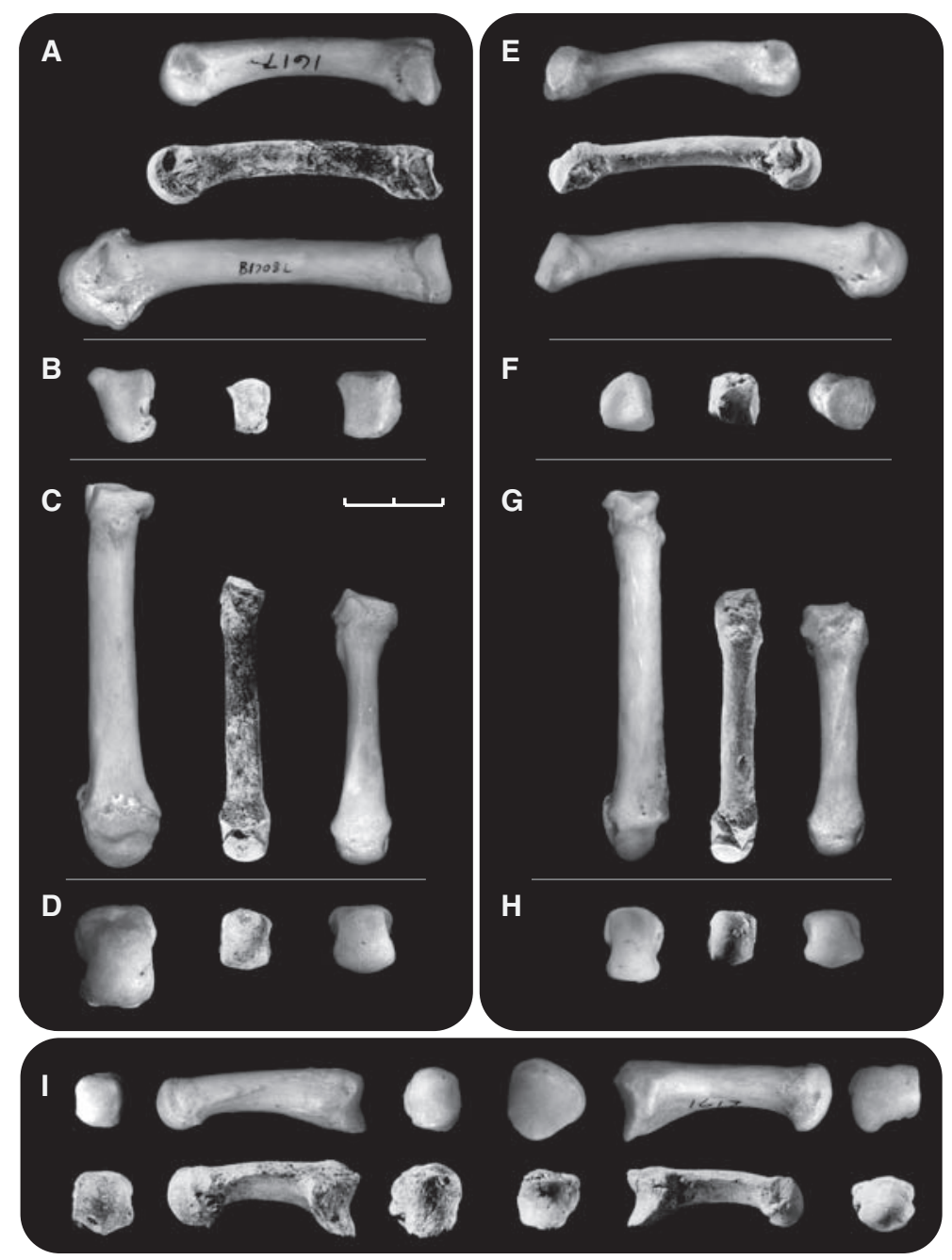

Fig. 2. Metacarpus of Ar. ramidus. (A to D) Left Mc4s of a modern human (CMNH-HT-1617), ARA-VP-7/2-G, and a chimpanzee (CMNH-B1708). Views include medial, proximal, dorsal, and distal. Note the very large notch on the lateral side of the base of the Pan specimen for transmission of its carpometacarpal ligament (a much smaller notch is present in the Ar. ramidus specimen) and the deep knuckle-walking sulcus on the head's dorsum (see also fig. S3). The latter is absent in hominids. (E to $\mathbf{H})$ Equivalent views of the left Mc5 of the same extant individuals and ARA-VP-6/500-036. The basal articular surface of the Ar. ramidus Mc5 is cylindrical and is continued well onto the shaft's dorsum, unlike either of the other specimens. Its form is almost identical to its homolog in Equatorius africanus. The Pan surface is virtually flat and nonmobile. (I) Mc1s of the same extant individuals (Pan, top left; Homo, top right), and two Ar. ramidus [ARA-VP-6/1638 (bottom right) and ARA-VP-6/500-015 (bottom left)]. Note the very broad Mc1 sellar bases in the hominids, demonstrating that palmar grasping is the probable antecedent condition for extant African apes and humans. This may have been lost in conjunction with apparent first-ray involution in apes $(71,72)$. Scale bar, $2 \mathrm{~cm}$. apes, the capitate head's surface usually bears a subchondral tidemark recording maximum dorsiflexion of the scaphoid, which engages only a portion of the capitate's dorsal surface (Figs. 3 and 4) (31-33). In Ar. ramidus, the scaphoid's distal articular margin is deeply concave. Here, dorsiflexion did not terminate on the capitate head, but continued onto the dorsal surface of the capitate's anatomical neck (Figs. 3 and 4). In Ar. ramidus, the scaphoid completely engulfed the head and thus entered hyperdorsiflexion, greatly enhancing its capacity for palmigrady at the midcarpal joint.

The palm of $A r$ ramidus is made relatively narrow by the dominance of its spherical lunate but mostly by its markedly narrow trapezoid (Fig. 3). The capitate head's palmar (and thereby eccentric) location may have limited midcarpal rotation in Ar. ramidus. However, its symmetric lunate, narrow trapezoid (figs. S26 to S29 and table S3), and more laterally facing scaphoid may have allowed greater compensatory radiocarpal axial rotation, and possibly greater radial deviation (see below), than in extant African apes and humans (in which the capitate's head is broad and less spherical). These changes appear to have occurred independently in humans for palmar grasping and in Pan and Gorilla for vertical climbing and knuckle-walking. If so, they have caused conflation of the functional and evolutionary history of the midcarpal joint [see, for example, $(32,33)]$.

Ar. ramidus establishes that the null hypothesis for evolution of the human hand must be that hominids have never had modern apelike CJCs or their attendant behaviors, in contrast to previous assumptions (32-34). Alteration of the primitive CJC in later hominids has been largely restricted to the following: (i) dorsal elevation of the capitate head; (ii) broadening of the capitate and trapezoid (for greater palmar span) (Fig. 3, figs. S28 and S29, and table S3); (iii) reduction of the surface relief in the Mc3capitate joint (thereby permitting greater kinematic compliance, probably initiated after the elimination of the forelimb from weight-bearing locomotion); and (iv) transfer of the os styloideum element from the capitate to the Mc3 $(28,29)$. Au. afarensis exhibits a more humanlike, albeit intermediate, condition. It shows evidence of all but the fourth of these shifts, each of which presumably facilitated nonlocomotor palmar grasping.

The much-discussed lateral orientation of the capitate's Mc2 and Mc3 facets [see review in (35)] in later hominids is probably now best viewed as a collateral pleiotropic effect [type $2 \mathrm{~A}$ (18)] of mediolateral expansion of the radial wrist associated with increased thenar size and robusticity, because both the trapezium and trapezoid are enlarged in Homo as compared with Ar. ramidus. The only possible kinematic significance of these minor variations of the capitate [that is, palmar cupping (24)] is most likely a consequence of cartilage modeling during on- 
togeny (type 4) or is simply inconsequential inherent variation (type $2 \mathrm{~B}$ and/or 5 ). The capitate's Mc2 and Mc3 angles are close to $90^{\circ}$ in both $A r$ ramidus and Au. anamensis (35). This

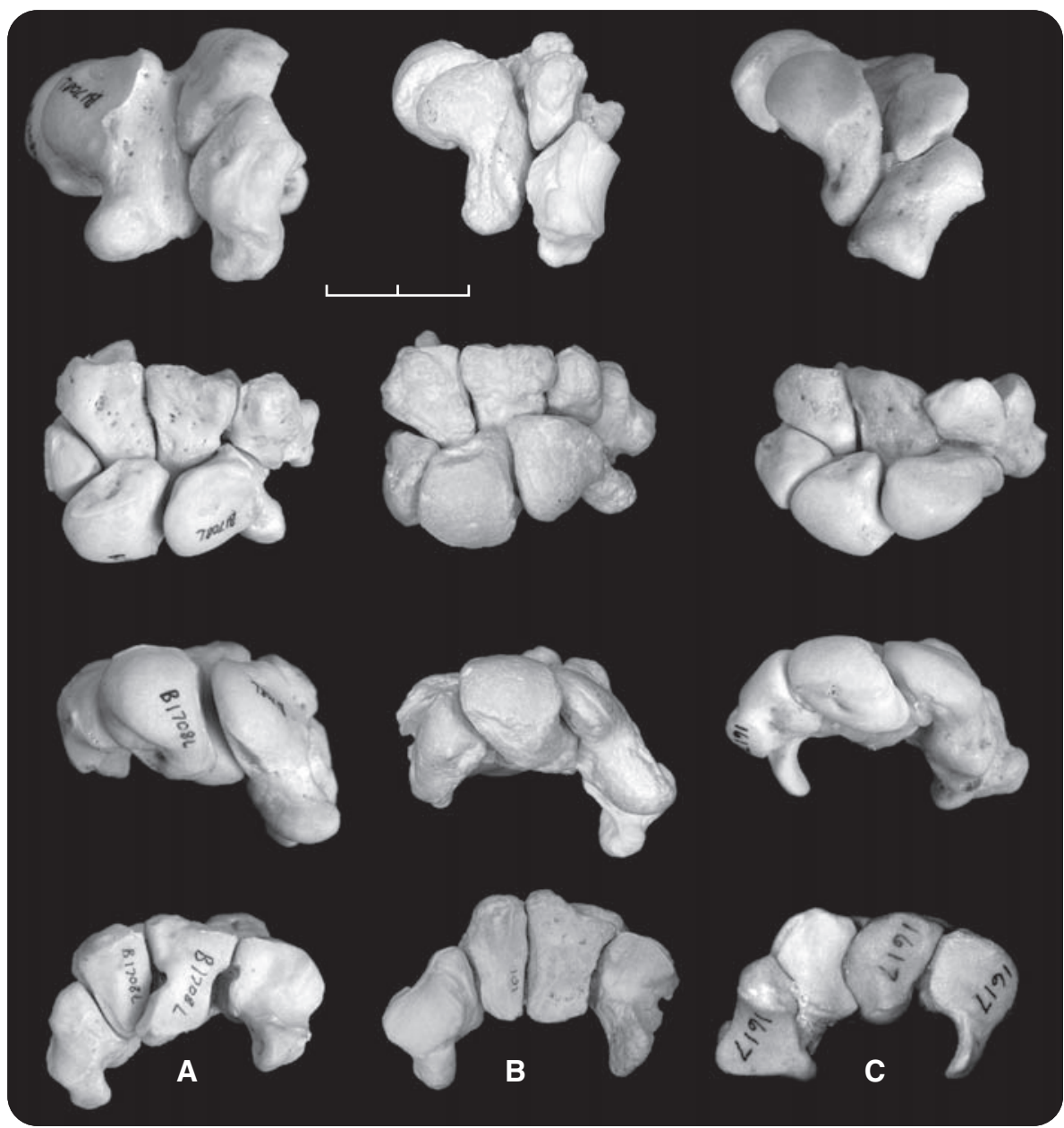

Fig. 3. Articulated wrists (left sides) of hominoids (no pisiform) in maximum dorsiflexion. (A) Pan (CMNH-B1708); (B) ARA-VP-6/500 (casts; the trapezium of ARA-VP-6/500 is a rapid prototyping model based on CT scan of the contralateral side); (C) Homo (CMNH-HT-1617). (Top row) Lateral view (Mc3 surface of capitate is vertical). The scaphoid's radial surface faces dorsolaterally in ARA-VP6/500. It faces slightly more proximal in Homo, but demonstrably more so in Pan. Its more limited articular extent in Pan allows enlarged radioscaphoid ligaments, which insert into its nonarticular area (see text, Fig. 4F, and fig. S30). (Second row) Dorsal view aligned with the capitate-hamate joint plane made vertical. The $A R A-V P-6 / 500$ scaphoid completely engulfs the capitate's head, advancing into a furrow formed between its articular neck and the proximal surface of the trapezoid. That of Pan articulates only with the more proximally directed capitate head. Both extant taxa have substantial mediolateral wrist expansion for palmar grasping in Homo and for knuckle-walking in Pan. Distally, there is a large styloid element on the ARA-VP-6/500 capitate [see text and (28)]. It has been transferred to the Mc3 in Homo, leaving behind a broad V-shaped recess. (Third row) Proximal view (plane of the capitate's Mc3 surface is vertical). The more proximally oriented radial surface of Pan is obvious, although that of Homo also faces less medially than that of Ar. ramidus (note the lunate's subchondral tidemark of maximum dorsiflexion superiorly as a slight dorsal ridge in Pan). (Fourth row) Distal view (the capitate-trapezoid joint plane is vertical). The capitate-trapezoid axis is simple in ARA-VP-6/500, whereas it has become medially concave in Homo, either facilitating or reflecting compliance within the carpus for palmar grasping. The distal carpal row is generally broadened in Homo. In Pan, the capitate's palmar portion has been extended distally, and large notches allow transit of its massive carpometacarpal ligaments (absent in hominids). Its hamate is broadened, with a more distally projecting and mediolaterally expanded hamulus. The distal face of the ARA-VP-6/500 capitate is a simple plane interrupted only by transverse palmar and dorsal furrows; its trapezoid is mediolaterally slender, whereas it has been mediolaterally expanded in Homo and Pan. Note the unusually large styloid element in Ar. ramidus [text and (28)].
In retrospect, it appears that subsequent to their last common ancestor with hominids, the Gorilla clade (and sometime thereafter the chimpanzee clade) faced an adaptive conundrum. Palmar conformity to substrates (primitive and retained in Ar. ramidus) is obviously beneficial to climbing. However, this imparts risk of injury to the hand from insufficient joint integrity and energy dissipation mechanisms that are apparently required during vertical climbing, suspension, and/or knuckle-walking. Only Pan appears to have eliminated substantial joint mobility in the Mc4- and Mc5-hamate joints entirely, but both African apes have evolved a sophisticated stabilizing mechanism in their CJCs.

Scaphoid morphology, ulnar retraction, and radiocarpal joint. The scaphoid of Ar. ramidus differs substantially from those of Old World monkeys, Proconsul, and other Miocene apes not only by the fusion of the os centrale, but by the elongation of its tuberosity and palmar deflection of its distal facet(s) for the trapezium and trapezoid (Fig. 3). These changes probably accompanied deepening of the carpal tunnel on the ulnar side of the wrist to prevent flexor tendon bowstringing by distal prolongation and increased prominence of the hamulus. Because the ulna was withdrawn to permit greater adduction, there was apparently a general deepening of the wrist's proximal transverse arch (25). This was probably also present in Pierolapithecus at $\sim 12.5$ million years ago (Ma) (36). Fusion of the os centrale may have been a collateral consequence [nonselected pleiotropic effect; type 2B (18)] of this reorganization. If so, ulnar retraction must have occurred independently in the ancestors of the hominid-African ape clade and Pierolapithecus, because the latter retained a separate os centrale (36). None of these advanced characters is present in Sivapithecus. Pongo also exhibits a form of ulnar retraction, but scaphoid-os centrale fusion is only rarely seen. Moreover, unlike the extant African apes, Pongo also exhibits substantial midcarpal rotation (37).

In extant African apes, the radiocarpal joint experiences large collision loads during knucklewalking. These are shared by the radiolunate and radioscaphoid articulations and account for a less proximodistal orientation of the scaphoid in African apes as compared with those of orangutans and Ar. ramidus. A markedly rugose and heavily buttressed scaphoid tubercle receives the hypertrophied styloscaphoid ligament in knuckle-walking apes, which restricts dorsiflexion that is imposed by ground reaction force (GRF) (38) and thereby maintains joint integrity and potentially contributes to the dissipation of collision forces. In addition, the ligamentous attachment area on the scaphoid's dorsum for the dorsal radiocarpal ligaments (that is, its nonarticular area; Figs. 3 and 4) is also enlarged in these apes.

The Ar ramidus scaphoid is unlike its African ape counterpart. Its nonarticular area is narrower, 
and its tuberosity is of small caliber, elongate, and relatively gracile, exhibiting virtually no expansion where it joins the bone's corpus. It would therefore be poorly suited to sustain large loads applied to its tuberosity by the styloscaphoid ligament. In humans, the scaphoid has been further reorganized to accommodate an enlarged trapezium. Ar. ramidus is near the mean of modern humans in an index reflecting the scaphoid's potential to sustain GRF (fig. S30), confirming that the Ar ramidus scaphoid lacks evidence of high-impact loading.
The Ar. ramidus lunate lies directly proximal to the head of the capitate, which accounts for its more spherical form than in humans or extant African apes. Lunate-hamate contact during dorsiflexion was minimal in Ar. ramidus, as in Old World monkeys, Proconsul (16), and humans (Fig. 3), whereas substantial lunate-hamate contact in Pan and Gorilla appears to be derived by capitate head expansion and scaphoid reorientation, probably in response to the collision loading of knuckle-walking, because Pongo lacks these features.
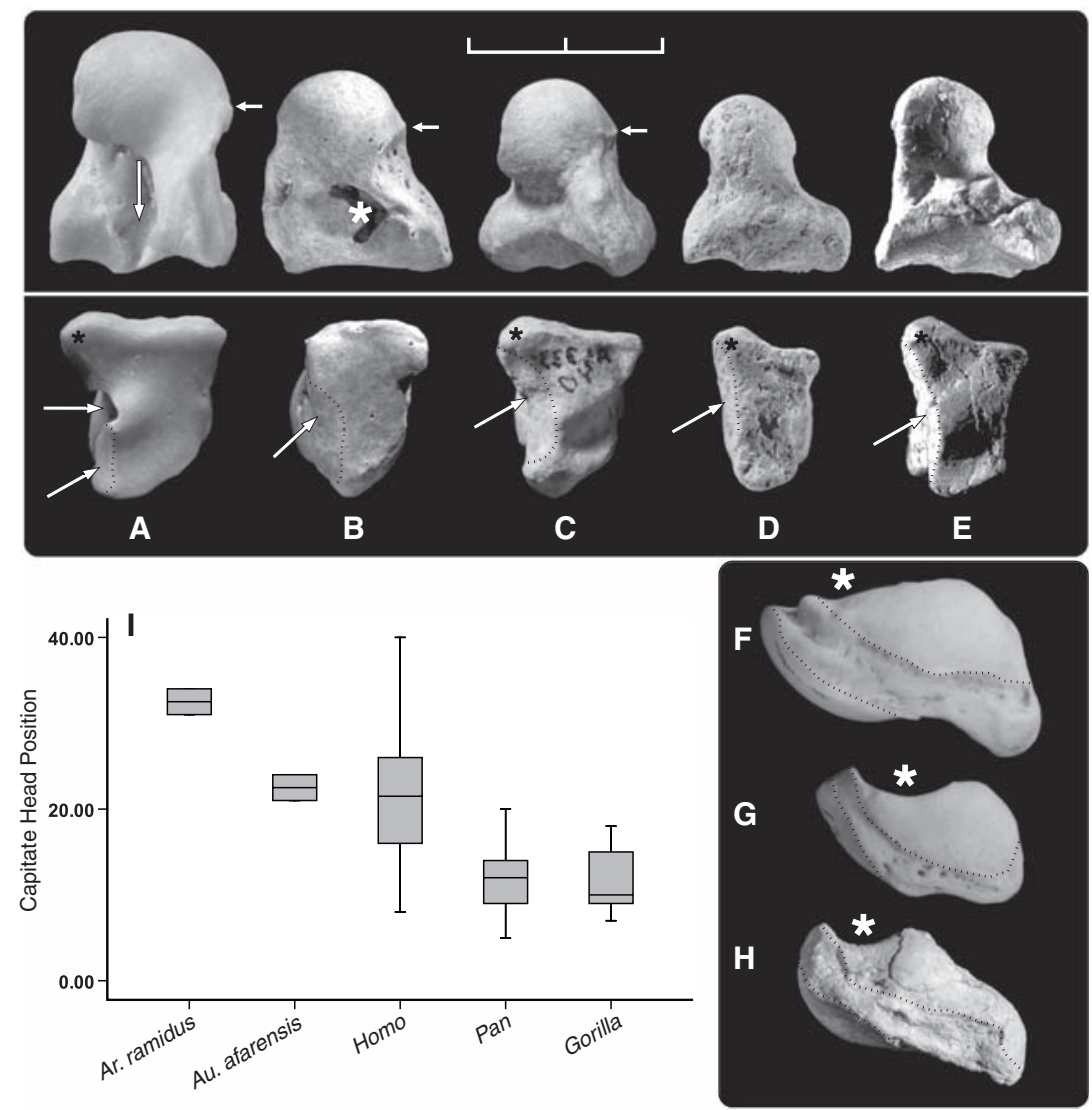

Fig. 4. Hominoid capitates and scaphoids. (A to E) Left capitate. Top, lateral (radial) view; bottom, distal capitate surfaces. (A) Pan (CMNH-B1718); (B) Homo (KSU-02234); (C) Au. afarensis [A.L. 333-40 (reversed)]; (D) Ar. ramidus (ARA-VP-7/2F); (E) Ar. ramidus (ARA-VP-6/500-058). (Bottom row) Hominid Mc3 surfaces exhibit only shallow dorsal and palmar transverse furrows. In Pan, the palmar capitate surface extends distally (toward the viewer), providing a buttress against the Mc2 [angled arrow in (A)] (see also Fig. $5 \mathrm{H}$ ) that prevents Mc3-capitate rotation. Angled white arrows point to broad, shallow, nonblocking Mc2 facets in hominids. The Homo and Au. afarensis capitates are mediolaterally expanded by a beveled Mc2 surface (dotted lines); it is much narrower in Ar. ramidus. A white asterisk marks a subchondral defect on (B) of no functional significance. The styloid element is marked by a black asterisk in each specimen except the human, in which it is instead fused to the Mc3 (Figs. 3 and 5). (Top row) Vertical arrow in (A) points to a large carpometacarpal ligament canal (as does horizontal arrow in bottom row). Hominids exhibit only uninterrupted cartilage surfaces. The Ar. ramidus capitate head is palmar, permitting marked dorsiflexion (see also Fig. 3). The head is more dorsal in apes and intermediate in Australopithecus and Homo. In Pan, the edge of the capitate's scaphoid articular surface is sharply delimited (horizontal arrow). In Au. afarensis and Homo, the head and neck blend imperceptibly, but a subchondral tidemark indicates maximum dorsiflexion (horizontal arrows). (F to H) Left scaphoids, trapezoid/trapezium surface faces superior; tuberosity is to the right, and the radial surface faces down to the left. (F) Pan (CMNH-1718), (G) Homo (KSU-12202), and (H) ARA-VP-6/500-062. Pan scaphoids often exhibit much shallower capitate notches (if present) (white asterisks) than hominids. Human scaphoid notch depth varies, but it is not typically as great as in $\operatorname{Ar}$. ramidus ( $n=2$; the human scaphoid notch shown is exceptionally deep). (I) Location of the capitate head in Ar. ramidus [for method, see (73)]. Scale bar, $2 \mathrm{~cm}$.

Lunate position in Ar. ramidus also accounts for the previously unexplained lunate/scaphoid facet area ratios on the distal radius of Australopithecus, which exceed those of both humans and extant African apes (table S4). However, it is now clear that scaphoid reorientation occurred in African apes for knuckle-walking and independently in humans for increased palmar span. The similar ratio in Ardipithecus and Australopithecus therefore represents the primitive condition for both hominid and extant African apes.

In summary, the hand of Ar. ramidus appears almost entirely primitive relative to the anatomical specializations seen in extant apes (for example, metacarpal elongation, elaboration of CJC articulations and ligaments, novel capitate geometry, reorientation of the scaphoid's radial surface, enlargement of the radioscaphoid ligament, relative diminution of the first ray, etc.). Ar. ramidus establishes that these changes in the ape hand are independent specializations for arboreal access and terrestrial travel (vertical climbing, forelimb suspension, knuckle-walking) and were apparently never established in hominids, which retained a more generalized, substrateconforming, grasping hand.

Radius and ulna. The Ar. ramidus sample includes a complete radius $[A R A-V P-6 / 500-039$; although damaged, its length is largely preserved (7)] and a second intact distal radius ( $A R A-V P$ $7 / 2-B)$. Both exhibit greater distal articular surface angulation relative to the shaft axis than do those other early hominids [as previously reported (39)], which is consistent with the scaphoid's more laterally facing radial facet (Fig. 3). This is now clearly identifiable as a primitive character, as previously surmised (35), and is not an adaptation to knuckle-walking [contrary to (40)]. Moreover, Ar. ramidus indicates that the radiocarpal joints of African apes and humans have become broadened mediolaterally in parallel, presumably for knucklewalking in the African apes and as a consequence of elaboration of the pollex for tool-using or -making and/or for extractive foraging in hominids. Scaphoid expansion and palmar broadening almost certainly underlie reduced radiocarpal joint angulation in later humans. Morphometrically based suppositions attributing these various characters to a history of knuckle-walking (40) or suspension in Australopithecus have been critiqued previously on theoretical grounds (41) and are now moot, because anatomical evidence indicates that $\mathrm{Ar}$. ramidus was never reliant on either.

The proximal ulna exhibits substantial differences among extant hominoids $(42,43)$. Some Miocene hominoid ulnae, as well as those of colobines, generally exhibit both long olecranons and anteriorly facing trochlear notches, a combination that is consistent with pronograde above-branch quadrupedality. Two proximal ulnae (ARA-VP-6/500-051 and $A R A-V P-7 / 2-C)$ were recovered at Aramis and show that the trochlear notch in Ar. ramidus faces anteriorly (table S5 and figs. S31 and S32). A cranially oriented trochlear notch with a retroflexed olec- 
Fig. 5. (A to D) Schematic of the articular geometry of the left CJC in hominoids. (A) Primitive planar condition of the CMC2 and CMC3 joints as seen in Ar. ramidus (and Homo). (B) The primitive C]C cannot resist shear or torsion (pronation/supination) except by distention by joint surface irregularities. (C) In African apes, the palmar capitate is insinuated distally into the interface between the Mc2 and Mc3, blocking CJC rotation (asterisk). (D) Dorsal schematic view of the Mc3-capitate portion of the C]C shown in (C). The dorsal and palmar portions of the capitate-Mc3 joint are oriented oppositely, creating a screw mechanism. Supination (26) of the

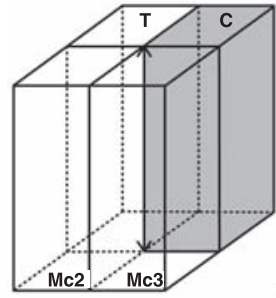

A
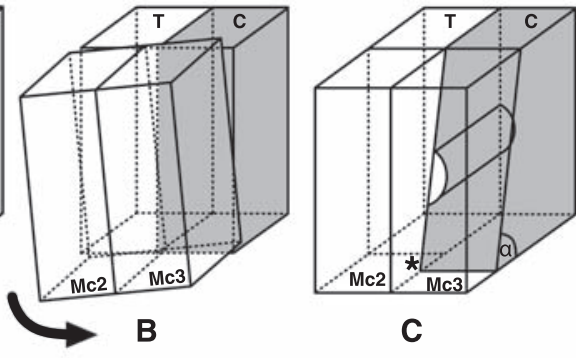

C

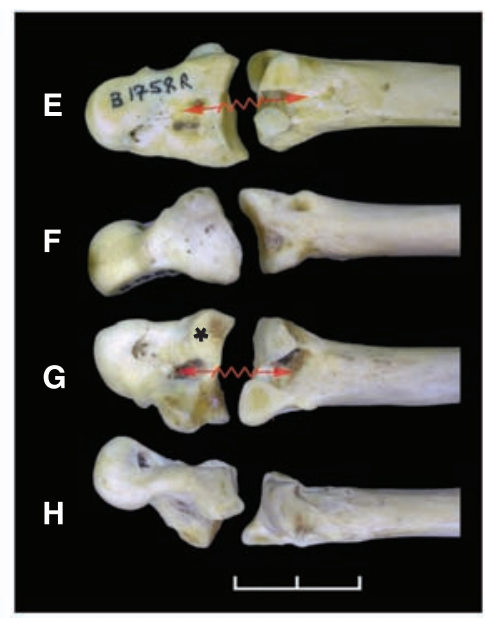

Mc3 results in joint distention resisted by its hypertrophied carpometacarpal ligaments (red springs) (the location and lateral orientation of the capitate's Mc2 facet are indicated by $\perp$ ). (E to $\mathbf{H})$ Medial, dorsal, lateral, and palmar views of an exploded right CMC3 joint in Pan CMNH-B1758. An asterisk indicates the capitate's palmar Mc2 facet, and red springs/arrows the carpometacarpal ligaments. The dorsal surface of each bone is angled opposite its palmar surface. This causes distention within the joint whenever it is supinated from a neutral (anatomical) position. ranon, as in African apes, enhances the triceps moment arm in full elbow extension. A more anteriorly facing notch favors triceps leverage at mid-flexion (42).

Although a substantially foreshortened olecranon is shared among hominids, Pan, and Gorilla, as well as with other middle and late Miocene hominoids that exhibit ulnar withdrawal at the wrist, a proximally oriented trochlear notch must be reflective of habitual suspension, because it is also found in Pongo. Retroflexion of the trochlear notch has, until now, been regarded as primitive, because the last common ancestor was presumed to have been adapted to some form of suspension. Instead, we conclude that an anteriorly facing notch is primitive, associated with an adaptation to careful climbing and bridging (44). Its presence in Ar. ramidus further supports the hypothesis that hominids have never been adapted to either suspension or vertical climbing and that human and ape proximal ulnar morphology converged for different reasons. The data for Ar. ramidus are consistent with arguments that early hominid ulnar morphology might reflect substantial manipulative skills (42), and that early hominids might have been involved in activities such as extractive foraging. In any case, ulnar morphology is congruent with transarticular force being generally greatest in full extension in both humans and great apes: during suspension in Pongo, suspension and knuckle-walking in extant African apes, and myriad possible manipulative activities unrelated to locomotion in hominids.

Another ulnar character in extant African apes, here termed the "flexor expansion," is a typically prominent, proximomedial enlargement adjacent to the posterior subcutaneous surface of the olecranon. This is created by the latter's juncture with a markedly deep excavation of the proximal-most origin of the deep digital flexor (fig. S33). When the ulna is viewed anteriorly, this medial projection reaches great prominence only in African apes (45) and is not present in $A r$ ramidus. It probably reflects the expansion of the mesenchymal territory of the flexor muscles' enthesis. This flexor expansion is derived and uniquely associable with knuckle-walking based on the role of the digital flexors (eccentric contraction and/or passive tension in their connective tissue capsules) and the enlarged relative muscle mass in Pan and Gorilla (38), as well as the tubercle's absence in orangutans and all hominids, including $A r$ r ramidus. The hypertrophy in extant African apes may have also been accompanied by myological reorganization in the forearm that resulted in gracilization or loss of the flexor pollicis longus tendon's attachment on the first ray.

Humerus. The Ar. ramidus humerus sample includes a well-preserved proximal humerus with shaft $(A R A-V P-7 / 2-A)$, a well-preserved humerus shaft (ARA-VP-1/4) (39) (fig. S34), and multiple distal humeral shafts lacking most or all of their distal articular surfaces (total $n=$ 7). Distal humeral morphology is largely conserved among hominoids, which vary only minimally in a variety of minor phenetic characters associated with full extension at the elbow (46), including a deep zona conoidea with a posteriorly extended lateral wall and a spherical capitulum with a short radius of curvature. Both are present in Ar. ramidus (ARA-VP-7/2-A) (47). The Ar. ramidus proximal humerus (39) exhibits equally typical hominid characters, including an elliptical head and shallow bicipital groove (48). It exhibits only minimal torsion (fig. S34).

The deltopectoral crest of $A R A-V P-1 / 4$ is elevated and rugose (39). The common assumption that this reflects differentially powerful arm musculature can be rejected for two reasons. First, the morphotype is shared among mod- ern humans, cercopithecoids, $P$. heseloni (14), Sivapithecus indicus (49), Ar. ramidus, and $A u$. afarensis. Second, the crest is consistently less developed in apes and virtually absent in brachiating gibbons. It is therefore a trait dictated primarily by positional information rather than loading (type 1 and/or 2) $(18,50,51)$. A rugose deltoid crest is clearly primitive (52), retained in hominids but substantially modified in suspensory, vertical-climbing, and knucklewalking apes - an observation made more than 75 years ago (53). A possible explanation of crest reduction in apes is that it reflects increased intermuscular fusion, as has occurred in gibbons (54), which also use suspension and exhibit almost no deltopectoral cortical surface manifestations. Deltopectoral morphology may therefore serve as a key indicator of locomotor habitus in other fossil hominoids.

Conclusions. The forelimb has played a definitive role in most chronicles of human evolution since Huxley's and Keith's original accounts $(55,56)$. Most recent narratives of its anatomical and behavioral evolution have emphasized a heritage of suspensory locomotion, vertical climbing, and knuckle-walking in the common ancestors that humans shared with extant African apes. Encouraged by human and chimpanzee genetic similarity and cladistic analyses, such views have come to dominate recent explications of early hominid evolution $(27,40)$, although alternative interpretations based on classical comparative anatomy have long differed $(3,57,58)$. Ar. ramidus now permits resolution of these controversies. It indicates that, although cranially, dentally, and postcranially substantially more primitive than Australopithecus, these known Late Miocene to Early Pliocene hominids probably all lacked the numerous, apparently derived, forelimb features of extant African apes. The most probable hypothesis to explain these observations is that hominids never passed through adaptive stages 
that relied on suspension, vertical climbing, or knuckle-walking. Further fossil remains from the Late Miocene, including those before and after the African ape-hominid phyletic divergences, will test this hypothesis derived from our analysis of Ar. ramidus.

\section{References and Notes}

1. G. Mivart, Proc. Zool. Soc. London 1873, 484 (1873).

2. C. Darwin, The Descent of Man, and Selection in Relation to Sex (John Murray, London, 1871).

3. W. L. Straus Jr., Q. Rev. Biol. 24, 200 (1949).

4. T. D. White et al., Science 326, 64 (2009).

5. C. O. Lovejoy, G. Suwa, L. Spurlock, B. Asfaw, T. D. White, Science 326, 71 (2009).

6. C. O. Lovejoy, B. Latimer, G. Suwa, B. Asfaw, T. D. White, Science 326, 72 (2009).

7. C. O. Lovejoy, G. Suwa, S. W. Simpson, J. H. Matternes, T. D. White, Science 326, 73 (2009).

8. D. R. Begun, J. Hum. Evol. 24, 373 (1993).

9. D. R. Begun, M. F. Teaford, A. Walker, J. Hum. Evol. 26, 89 (1994).

10. S. Ward, B. Brown, A. Hill, J. Kelley, W. Downs, Science 285, 1382 (1999).

11. S. Almecija, D. M. Alba, S. Moya-Sola, M. Kohler, Proc. Biol. Sci. 274, 2375 (2007).

12. M. E. Bush, C. O. Lovejoy, D. C. Johanson, Y. Coppens, Am. J. Phys. Anthropol. 57, 651 (1982).

13. Apparently stemming from (33), the morphology of the Mc5-hamate joint in Au. afarensis has been mischaracterized. Its Mc5-hamate joint is fundamentally similar in both structure and function to that of $H$. sapiens, save for the effects of the latter's dramatic palmar rotation of the hamulus. The original observations of these specimens were that the four preserved Mc5 bases were "convex" (that is, condyloid) and lacked "true articulation with the hamulus" [(12), p. 659]. Thus, the statement that it "is obvious that the bases of the fourth and fifth metacarpals articulated with the hamate [in afarensis] as in Pan" [(33), p. 178] is unsubstantiated and incorrect.

14. J. R. Napier, P. R. Davis, Fossil Mammals of Africa No. 16 (British Museum of Natural History, London, 1959).

15. K. C. Beard, M. F. Teaford, A. Walker, Folia Primatol. (Basel) 47, 97 (1986).

16. K. C. Beard, M. F. Teaford, A. Walker, in Hands of Primates, H. Preuschoft, D. J. Chivers, Eds. (SpringerVerlag, Vienna, 1993), p. 387.

17. A. C. Walker, M. Pickford, in New Interpretations of Ape and Human Ancestry, R. L. Ciochon, R. S. Corruccini, Eds. (Plenum, New York, 1983) p. 325.

18. The trait nomenclature system used here is taken from $(50,59)$ and is briefly as follows [for more complete explanations, see (4)]. Type 1: traits whose morphogenesis is the direct consequence of pattern formation; usually (but not always) subjected to direct selection. Type 2: traits that are genetic but are pleiotropic to, or result from, hitchhiking on type 1 traits and are not themselves subject to selection. 2A: Parent type 1 is inferred to be under selection; its secondary effects are not. 2B: Neither parent trait nor its derivative is inferred to be under selection (rare). Type 3: resulting from a systemic growth factor. Type 4: epigenetic consequence of osteochondral remodeling and/or response to environmental stimuli; that is, not heritable but useful in interpreting behavior. Type 5 : developmentally similar to type 4, but functionally uninformative.

19. C. F. Spoor, P. Y. Sondaar, S. T. Hussain, J. Hum. Evol. 21, 413 (1991).

20. Whereas the digits of apes have an "astonishing interdigital versatility" not seen in humans, only humans have an entirely independent flexor pollicis longus combined with specialized "digital pairing" of flexor tendons 3 and 4 and 2 and 5 . These provide the uniquely human "cylindrical grip," which we regard as the likely selective target of changes in the hands of early hominids; that is, "palmar grasping." In humans, digits 2 and 3 oppose the expanded tip of the thumb, and 4 and 5 the thenar eminence, providing afferent/efferent "information on shape, consistency, and surface qualities" [(60), p. 331]. That the necessary proportional and neural changes were eventually achieved in Homo is adequate evidence that although obviously still lacking in the hands of $A u$. afarensis, early recognizable manual changes in this taxon (for example, an increase in palmar breadth and greater length of ray 1 ) indicate a selective target of hand function similar to that eventually achieved in Homo.

21. B. Asfaw et al., Science 284, 629 (1999).

22. M. W. Marzke, M. M. Shrewsbury, J. Hum. Evol. 51, 213 (2006).

23. M. M. Shrewsbury, M. W. Marzke, R. L. Linscheid, S. P. Reece, Am. J. Phys. Anthropol. 121, 30 (2003).

24. F. J. Bejjani, J. M. F. Landsmeer, in Basic Biomechanics of the Musculoskeletal System, M. Nordin, V. H. Frankel, Eds. (Lea \& Febiger, Philadelphia, 1989), p. 275.

25. M. Baratz, A. D. Watson, J. E. Imbriglia, Orthopaedic Surgery: The Essentials (Thieme, New York, 1999).

26. A "screw"-type ligament tensing mechanism must be unidirectional, because any opposite rotation could potentially cause cartilage damage by shear generated during compression. This is prevented by the novel abutment of the capitate's palmar process against the medial face of the Mc2.

27. J. G. Fleagle et al., Symp. Zool. Soc. London 48, 359 (1981).

28. The styloid process of the human Mc3 is almost certainly the result of embryogenetic transfer of a styloid anlaga from the capitate to the Mc3. A separate os styloideum is frequently found in humans or is adherent to the capitate or trapezoid [total: $6 \%$ of cases (61)]. Indeed, an os styloideum is so frequently encountered that it was at one time considered the "ninth carpal" (62).

29. Mobility in the Mc4- and Mc5-hamate joints has been further enhanced in humans by palmar angulation of the hamulus, presumably after a long period of freedom from the selective rigors of locomotion. Moreover, ulnar angulation of the medial metacarpals cannot have the same importance in apes as in humans, because the African ape first ray has undergone involution rather than hypertrophy.

30. The capitate of $P$. heseloni shows a slight depression that may foreshadow the eventually dramatic relocation of the head that is present in Ar. ramidus [see especially $(15,16)]$.

31. Capitate waisting has been argued to facilitate midcarpal locking and to be a suspensory adaptation in apes [and early hominids $(32,33)]$. Frictionless synovial diarthroses promote motion, and joints do not normally lock because it is hazardous to their structure (41). In most joints, stability is instead almost entirely the responsibility of ligaments and soft tissues [contractile and connective tissue (noncontractile) components of the joint's surrounding muscles (63)]. If these did not arrest motion at and near full midcarpal dorsiflexion (64), the capitate's precipitous distal expansion would habitually strain the scaphoid-lunate syndesmosis, leading to its deterioration and instability. We agree with recent observations that midcarpal structures differ substantially in Gorilla and Pan (65); however, knuckle-walking functions assigned to minor surface topographic fluctuations of the scaphoid, capitate, and hamate reflect only the ontogenetic interplay of cartilage modeling, positional information, and the stabilizing soft tissues surrounding these joints. Mere concavities (or ridges) on synovial surfaces are almost never able to restrict motion and are more likely to merely reflect its limits as dictated by the joint's surrounding soft tissues (63). These features reflect the joint's likely kinematics, but little about its kinetics.

32. 0. J. Lewis, Nature 230, 577 (1971).

33. O. J. Lewis, Functional Morphology of the Evolving Hand and Foot (Clarendon Press, Oxford, 1989).

34. M. G. Leakey, C. S. Feibel, I. McDougall, C. Ward, A. Walker, Nature 393, 62 (1998).

35. C. V. Ward, M. G. Leakey, A. Walker, J. Hum. Evol. 41, 255 (2001)

36. S. Moya-Sola, M. Kohler, D. M. Alba, I. Casanovas-Vilar, J. Galindo, Science 306, 1339 (2004).

37. F. A. J. Jenkins, J. Zool. Soc. London 48, 429 (1981).
38. R. H. Tuttle, thesis, University of California, Berkeley, CA (1965).

39. T. D. White, G. Suwa, B. Asfaw, Nature 371, 306 (1994).

40. B. G. Richmond, D. S. Strait, Nature 404, 382 (2000).

41. C. O. Lovejoy, K. G. Heiple, R. S. Meindl, Nature 410, 325 (2001).

42. M. S. Drapeau, C. V. Ward, W. H. Kimbel, D. C. Johanson, Y. Rak, J. Hum. Evol. 48, 593 (2005).

43. M. S. Drapeau, Am. J. Phys. Anthropol. 124, 297 (2004).

44. M. Cartmill, K. Milton, Am. J. Phys. Anthropol. 47, 249 (1977).

45. L. C. Aiello, B. Wood, C. Key, M. Lewis, Am. J. Phys. Anthropol. 109, 89 (1999).

46. This is expected given the following: (i) advanced antebrachial anatomy with full ulnar withdrawal $(32,33,44)$ and pronation/supination were already present in Pierolapithecus at $\sim 12.5 \mathrm{Ma}$ (36), and (ii) the primary differences in elbow morphology take place in the ulna, rather than the humerus. A parallel relationship occurs in the joint's hindlimb counterpart: The femur contains most functional information about kinematic behavior in the knee; the tibia relatively little.

47. Some have argued that functionally and phylogenetically meaningful distinctions can be made among Pliocene hominid humeri, and that the Kanapoi distal humerus has affinities with Homo (66), whereas other Pliocene materials of Australopithecus and Ar. ramidus represent primitive hominid or ape branches cladistically outside of Orrorin $(66,67)$. However, two morphometric studies of Pliocene hominid distal humeri $(68,69)$ have independently confirmed our visual assessments that these humeri cannot be segregated into meaningful morphotypes, thus leaving no quantifiable basis for such assertions.

48. M. Pickford, D. C. Johanson, C. O. Lovejoy, T. D. White, J. L. Aronson, Am. J. Phys. Anthropol. 60, 337 (1983).

49. J. Kelley, in The Primate Fossil Record, W. C. Hartwig, Ed. (Cambridge Univ. Press, Cambridge, 2002), pp. 369-384.

50. C. O. Lovejoy, M. J. Cohn, in Development, Growth, and Evolution, P. O'Higgens, M. ]. Cohn, Eds. (Academic Press, London, 2000), pp. 41-55.

51. A. Zumwalt, J. Exp. Biol. 209, 444 (2006).

52. B. Benefit, M. McCrossin, Annu. Rev. Anthropol. 24, 237 (1995).

53. "A comparison with the humerus of the anthropoid [apes] shows that the Sinanthropus humerus is as different from it as the humerus of modern man. Not in one single feature does Sinanthropus reveal a true anthropoid character . . . [T] he deltoid tuberosity . . . is very poorly developed in all three great apes. . . . It is, therefore, all the more surprising that the tuberosity is very pronounced in [Macaca and Cynocephalus]. . . . If, therefore, the peculiar shape of the deltoid tuberosity in the Sinanthropus humerus is to be interpreted as a simian character, it is one that must be traced back to a pre-anthropoid stage" [(70), p. 60].

54. A. B. Howell, W. H. Straus, Proc. U. S. Natl. Mus. 80, 1 (1938).

55. T. H. Huxley, Evidence As to Man's Place in Nature (Williams \& Norgate, London, 1863).

56. A. Keith, Br. Med. J. 1, 788 (1912).

57. A. H. Schultz, Q. Rev. Biol. 11, 259 (1936).

58. Straus observed that "[t]here can be no reasonable doubt that a long thumb (relative to the other fingers) is a generalized pithecoid character, or that its marked relative reduction in such animals as the anthropoids, some of the Semnopithecinae, and certain platyrrhines, is an extreme specialization correlated with addiction to brachiation" (3). He observed that human thumb musculature is probably primitive because of the following: (i) "a morphologically complete and functional long flexor to the thumb... is normally absent in orangs, and present in less than half of African apes but is constant in prosimians, platyrrhines . . . Cercopithecinae, the Hylobatidae, and man" [(3), p. 87]; and (ii) "The short, intrinsic, volar muscles of the thumb [which are either weakly developed or absent in the apes] are regularly welldeveloped in man, the Hylobatidae, the Old World monkeys (except Colobus), the New World monkeys (except Ateles), and the prosimians" [(3), p. 87]. 
59. C. O. Lovejoy, R. S. Meindl, ]. C. Ohman, K. G. Heiple, T. D. White, Am. ]. Phys. Anthropol. 119, 97 (2002).

60. J. M. F. Landsmeer, in Hands of Primates, H. Preuschoft, D. J. Chivers, Eds. (Springer-Verlag, Vienna, Austria, 1993), pp. 323-333.

61. R. O'Rahilly, J. Bone Jt. Surg. 35-A, 626 (1953).

62. E. A. Zimmer, S. P. Wilk, Borderlands of the Normal and Early Pathologic in Skeletal Roentgenology (Grune \& Stratton, New York, 1968).

63. A. H. Burstein, T. W. Wright, Fundamentals of Orthopaedic Biomechanics (Williams \& Wilkins, Baltimore, 1994).

64. W. L. Straus, Am. J. Phys. Anthropol. 27, 199 (1940).

65. T. L. Kivell, D. Schmitt, Proc. Natl. Acad. Sci. U.S.A. 106, 14241 (2009).

66. B. Senut, S. Afr. J. Sci. 92, 165 (1996).

67. B. Senut et al., Sci. Paris. 332, 137 (2001).

68. M. R. Lague, W. L. Jungers, Am. J. Phys. Anthropol. 101 401 (1996).

69. A. M. Bacon, Am. J. Phys. Anthropol. 111, 479 (2000).

70. F. Weidenreich, Palaeontologia Sinica, Whole Series No. 116; New Series D, No. 5 (Geological Survey of China, Peking, China, 1941).
71. C. O. Lovejoy, M. J. Cohn, T. D. White, Proc. Natl. Acad. Sci. U.S.A. 96, 13247 (1999)

72. P. L. Reno et al., J. Exp. Zool. B Mol. Dev. Evol. 310, 240 (2008).

73. Photographs were taken normal to the capitate's lateral surface, and a vertical tangent was inscribed along the palmar and dorsal surfaces of its Mc3 facet.

Three perpendiculars were then erected to this vertical tangent: (i) a horizontal tangent (at the bottom) to the most palmar point on the capitate; (ii) a horizontal tangent at the top to the most dorsal point on the capitate, and (iii) a horizontal tangent to the dorsalmost point on the articular surface of the capitate head. The distance between tangents ii and iii was then normalized by the total distance between tangents $i$ and ii.

74. Supported by NSF (this material is based on work supported by grant numbers $8210897,9318698,9512534$, 9632389, 9729060, 9910344, and 0321893 HOMINID-RHOI), and the Japan Society for the Promotion of Science. We thank the Ministry of Tourism and Culture, the Authority for Research and Conservation of the Cultural Heritage, and the National Museum of Ethiopia for permissions and facilitation; the Afar Regional
Government, the Afar people of the Middle Awash, and many other field workers for contributing directly to the data; the following institutions and staff for access to comparative materials: National Museum of Ethiopia, National Museums of Kenya, Transvaal Museum South Africa, Cleveland Museum of Natural History, Royal Museum of Central Africa Tervuren, and the University of California at Berkeley Human Evolution Research Center; D. Kubo and H. Fukase for assistance in computerized tomography scanning and R. T. Kono for the rapid prototyping model; R. Meindl for statistical advice and assistance; P. L. Reno, M. A. Serrat,

M. A. McCollum, M. Selby, A. Ruth, L. Jellema, D. DeGusta, and B. A. Rosenman for aid in data collection and exceptionally helpful discussions; and K. Brudvik, H. Gilbert, and J. Carlson for figure preparation.

\section{Supporting Online Material}

www.sciencemag.org/cgi/content/full/326/5949/70/DC1

Figs. S1 to S34

Tables S1 to S5

References

4 May 2009; accepted 18 August 2009

10.1126/science. 1175827 